\section{Embrittlement of uterus after uterine artery embolization: a case of uterine perforation}

\author{
Yasuko Sano, Jun Takeda, Keiji Kuroda, Shintaro Makino, \\ Atsuo Itakura, Satoru Takeda
}

Department of Obstetrics and Gynecology, Juntendo University Faculty of Medicine, Tokyo, Japan
Reprint request to:

Jun Takeda, M.D., Ph.D., Juntendo University Faculty of Medicine, 2-1-1 Hongo, Bunkyo-ku, Tokyo 113-8421, Japan.

E-mail: jtakeda@juntendo.ac.jp

\section{Key words:}

intrauterine synechia, magnetic resonance imaging, uterine artery embolization, side effect, uterine necrosis

Received: March 28, 2016

Revised: April 8, 2016

Accepted: June 6, 2016

DOI:10.14390/jsshp.HRP2015-017

Uterine artery embolization (UAE) is a common treatment for postpartum hemorrhage, however, the influences on uterus have yet to be uncertain. Here, we present a case of uterine embrittlement and perforation after UAE. A 30-year-old primipara received UAE for postpartum hemorrhage. Enhanced magnetic resonance imaging (MRI) after UAE showed lack of enhancement on uterine fundus. She complained hypomenorrhea after UAE, thus hysteroscopy was performed for intrauterine synechia. During the surgery, uterine perforation, which identical with the ischemic part on MRI, was found. It was repaired laparoscopically together with vulnerable surrounded area. With the case, because UAE tend to choose for woman who desire for the next pregnancy, we recommend that UAE should be limited and performed only for cases that could not accomplished hemostasis even with other methods. When UAE was chosen reluctantly, uterine myometrium ischemia should be monitored by contrast enhanced MRI.

\section{Introduction}

Uterine artery embolization (UAE) is a common and minimally invasive treatment for postpartum hemorrhage $(\mathrm{PPH}) .{ }^{1)}$ It has become an alternative for hysterectomy due to uncontrolled postpartum hemorrhage, especially for women who have desire for subsequent pregnancy. UAE has also been used for gynecological disorders such as uterine fibroids as well. ${ }^{2)}$ However, UAE has a possibility to lead to severe side effects in low frequency, such as infertility, uterine myometrium necrosis and in consequence uterine rupture of subsequent pregnancy. ${ }^{3-8)}$ Here, we present a case of uterine embrittlement in a patient who had UAE for PPH.

\section{Case report}

A 30-year-old gravida 0, para 0 woman delivered a baby by vacuum extraction at private clinic. Postpartum hemorrhage became $1,940 \mathrm{ml}$ and she was transferred to our hospital for PPH. The vital signs at our hospital were as follows; heart rate $123 / \mathrm{min}$, blood pressure $126 / 75 \mathrm{mmHg}$, thus shock index was nearly one. Blood examination revealed $3.3 \mathrm{~g} / \mathrm{dl}$ of low hemoglobin. Left side of the vulva was swelled and huge hematoma was found inside the vaginal wall. However, we couldn't detect where the bleeding was come from. Japanese Society of interventional Radiology recommends in guideline that embolization of bilateral uterine arteries in case of bleeding of unknown source for PPH, therefore, UAE was chosen for hemostasis. Bilateral internal iliac angiography via a right inguinal approach showed no extravasation from either uterine artery, however, as the hemorrhage persisted, Gelpart (Nippon Kayaku Co., Ltd., Tokyo, Japan) a soluble embolization agent, was injected into bilateral uterine artery (left: $240 \mathrm{mg}$, right: $160 \mathrm{mg}$ ) until the bleeding ceased. Fever over 39 degrees Celsius and inflammatory reactions of $\mathrm{C}$ reactive protein up to 20 were observed within 10 days post operation. Uterine infection after UAE was suspected, however, no evidence including positive vaginal culture and high procalcitonin were found to support it. Conservative therapy using antibiotics seemed effective and she was discharged 40 days after UAE.

Contrast enhanced MRI performed on day 13 post UAE showed lack of enhancement on majority of 


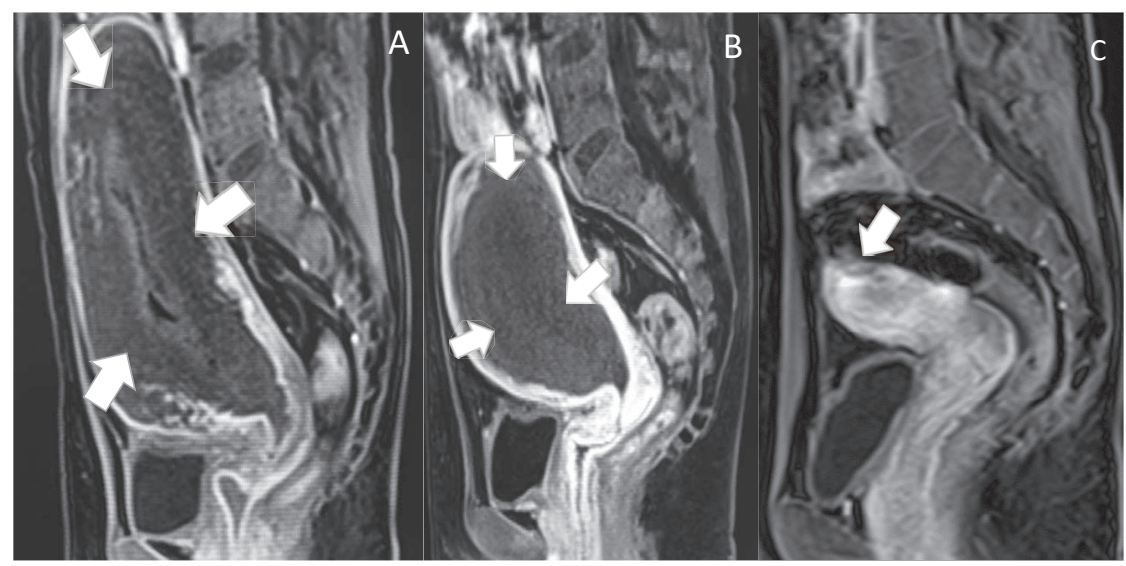

Figure 1. The images of contrast enhanced MRI.

Lack of enhancement on majority of uterine myometrium was found on contrast enhanced MRI on day 13 post uterine artery embolism (UAE) (A). The enhanced area was gradually increasing on day 58 post UAE, but still majority of uterine myometrium was lacked of enhancement (B). Contrast enhanced MRI performed on day 243 post UAE showed recovered of enhancement on most of the myometrium, however, still poorly enhanced area remained at the mid posterior wall of uterine fundus (C). The arrows showed the poorly enhanced area.

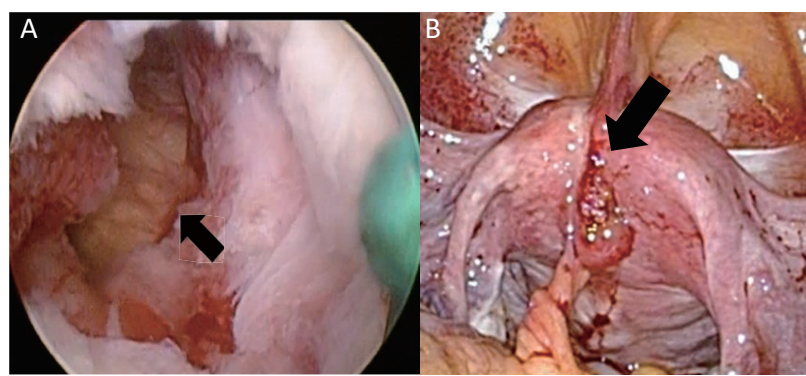

Figure 2. The images of hysteroscopy and laparoscopy. Hysteroscopy observation after uterine cervix dilation showed omentum majus in the uterine cavity (A), and uterine perforation at the mid posterior wall of uterine fundus was confirmed by laparoscopic observation (B).

uterine myometrium (Figure 1A). The enhanced area on MRI was gradually increasing on day 58 post UAE, but still majority of uterine myometrium were lacked of enhancement (Figure 1B). MRI performed on day 243 post UAE showed recovered of enhancement on majority of myometrium, however, still poorly enhanced area remained at the mid posterior wall of uterine fundus (Figure 1C).

Menstruation resumed spontaneously 13 months after the UAE, however, she complained decreased amount of menstruation. Thus, she consulted us again for the next pregnancy. Transvaginal ultrasonography revealed obscure image of the endometrium, thus hysterosalpingography was performed. Contract agent flow were hard to spread into the uterus and she was diagnosed as Asherman's syndrome. Operative hysteroscopy was performed to treat Asherman's syndrome on 16 months post UAE. Hysteroscopy observation after uterine cervix dilation showed omentum majus in the uterine cavity (Figure 2A), and uterine perforation at the mid posterior wall of uterine fundus was confirmed by laparoscopic observation (Figure 2B). Although fragile myometrium and easily teared of the myometrium by the handling of needles were found, uterine wall was repaired laparoscopically.

\section{Discussion}

UAE is well known for effective therapy for PPH and its success rate is approximately $90 \%$ in the previous report. ${ }^{1)}$ Several reports showed series of woman who had successful deliveries after UAE.,4) Particularly for women who wish for a subsequent pregnancy, UAE has been conformed to the needs to preserve fertility and superior alternative approach to hysterectomy. However, as a complication, cases of uterine necrosis after UAE has been reported. ${ }^{5-8)}$ Poujade et al. reviewed 19 cases of uterine necrosis, which were all diagnosed with resected uterus, and mentioned that one of the main symptoms of uterine necrosis was high fever. ${ }^{6}$ The symptom was matched with our cases and possibility of uterine necrosis was remained, not hysterectomy but conservatory therapy was chosen with our case. The reasons we chose to preserve uterus were the patient strongly desired for a subsequent pregnancy and also the situation that fever and inflammatory reactions gradually recovered allowed to do so. Although whether the ischemic uterus, possibly necrotic uterus, remains fertility or not, much effort 
should be given to preserve uterus. As a result, our case succeeded to preserve uterus, which enabled us to know how the uterus ischemia shift for long term. Mid posterior wall of the uterus remained ischemic region, however, the enhanced area on MRI was gradually increasing and enlarged uterus started to be contracted to its original condition, suggesting that usefulness of contrast enhanced MRI for evaluating not only for acute necrosis but also for chronic ischemia.

Previously, we had reported a case of spontaneous uterine rupture at subsequent pregnancy after UAE. ${ }^{8)}$ In that case, uterine necrosis observed exactly same region with our case. MRI showed similar pattern as well that the image on day 125 post UAE showed poorly enhanced areas in the mid posterior wall of uterine fundus. The site of perforation and rupture, present case and previous case respectively, were identical to the poorly enhanced region on contrast enhanced MRI. Previously, we had speculated that mid posterior wall of the uterine fundus was watershed region between the terminal arterioles of the left and right uterine arteries, and had discussed that even with the use of soluble embolization material and in the face of enhanced uterine blood flow, including via ovarian arteries, UAE in early pregnancy could lead to uterine necrosis. Our present case pushes the speculation. The difference between our previous report and the present case was gestational age at UAE. We performed UAE within 12 hours after spontaneous term delivery in this case. Uterine blood flow via collateral arteries seemed much more than early pregnancy, nevertheless, it could not prevent the uterine necrosis. Therefore, because UAE tend to choose for a woman who desire for a next pregnancy, we recommend that UAE should be limited and performed only for the cases that could not accomplished hemostasis even with other methods such as compression sutures, ${ }^{9}$ balloon tamponade ${ }^{10)}$ and so on. When those attempts had failed, the situation would be suitable for UAE. When UAE was chosen, uterine myometrium ischemia should be monitored by contrast enhanced MRI.

\section{Acknowledgements}

None.

\section{Conflict of interest}

None.

\section{References}

1. Ratnam LA, Gibson M, Sandhu C, Torrie P, Chandraharan E, Belli AM. Transcatheter pelvic arterial embolisation for control of obstetric and gynaecological haemorrhage. J Obstet Gynaecol. 2008; 28: 573-579.

2. Bradley LD. Uterine fibroid embolization: A viable alternative to hesterectomy. Am J Obstet Gynecol. 2009; 201: 127-135.

3. Sentilhes L, Gromez A, Clavier E, Resch B, Verspyck E, Marpeau L. Fertility and pregnancy following pelvic arterial embolisation for postpartum haemorrhage. BJOG. 2010; 117: 84-93.

4. Mclucas B, Voorhees iii WD, Elliott S. Fertility after uterine artery embolization: a review. Minim Invasive Ther Allied Technol. 2016; 25: $1-7$.

5. Poujade O, Daher A, Ballout El, et al. Uterine necrosis following pelvic arterial embolisation for postpartum haemorrhage: case report. J Gynecol Obstet Biol Reprod (Paris). 2012; 41: 584-586.

6. Poujade O, Ceccaldi PF, Davitian C, et al. Uterine necrosis following pelvic arterial embolization for post-partum hemorrhage: review of the literature. Eur J Obstet Gynecol Reprod Biol. 2013; 170: 309-314.

7. Gabriel H, Pinto CM, Kumar M, et al. MRI detection of uterine necrosis after uterine artery embolization for fibroids. AJR Am J Roentgenol. 2004; 183: 733-736.

8. Takeda J, Makino S, Ota A, Tawada T, Mitsuhashi N, Takeda S. Spontaneous uterine rupture at 32 weeks of gestation after previous uterine artery embolization. J Obstet Gynaecol Res. 2014; 40: 243-246.

9. Makino S, Tanaka T, Yorifuji T, Koshiishi T, Sugimura M, Takeda S. Double vertical compression sutures: A novel conservative approach to managing post-partum haemorrhage due to placenta praevia and atonic bleeding. Aust N Z J Obstet Gynaecol. 2012; 52: $290-292$.

10. Georgiou C. Balloon tamponade in the management of postpartum haemorrhage: a review. BJOG. 2009; 116: 748-757. 